Ego Depletion Reduces Attention Control: Evidence from Two High-Powered Preregistered Experiments

Katie E. Garrison, Anna J. Finley, \& Brandon J. Schmeichel Texas A\&M University

Author Note

In Press: Personality and Social Psychology Bulletin

Correspondence concerning this article should be addressed to Brandon Schmeichel, Department of Psychology, Texas A\&M University, 4235 TAMU, College Station, TX 77840. Contact: bjschmeichel@gmail.com

Word count: 10,287 


\begin{abstract}
Two preregistered experiments with over 1000 participants in total found evidence of an ego depletion effect on attention control. Participants who exercised self-control on a writing task went on to make more errors on Stroop tasks (Experiment 1) and the Attention Network Test (Experiment 2) compared to participants who did not exercise self-control on the initial writing task. The depletion effect on response times was non-significant. A mini meta-analysis of the two experiments found a small $(d=0.20)$ but significant increase in error rates in the controlled writing condition, thereby providing evidence of poorer attention control under ego depletion. These results, which emerged from preregistered experiments in large samples of participants, represent some of the most rigorous evidence yet of the ego depletion effect.
\end{abstract}

Keywords: Ego depletion, self-control, attention control, preregistration 


\section{Ego Depletion Reduces Attention Control: Evidence from Two High-Powered Preregistered Experiments}

Overriding impulses or otherwise controlling one's thoughts, behaviors, and emotions is crucial for human functioning and wellbeing (Mischel, Shoda, \& Peake, 1988; Tangney, Baumeister, \& Boone, 2004). One prominent theoretical model of self-control is the resource model, which holds that self-control relies on an inner energy that depletes with use (Muraven \& Baumeister, 2000). In this view, a temporarily depleted capacity for control (i.e., ego depletion) is a major cause of self-control failure.

Despite over 200 published studies reporting evidence in support of the resource model, the behavioral aftereffects of self-control remain in doubt. A handful of published studies have reported null effects, including a high-powered replication study conducted across 23 laboratories (Hagger et al., 2016), and much of the prior evidence for ego depletion comes from studies with relatively small samples (i.e., typically no more than 40 participants per experimental condition). A recent meta-analysis attempted to account for publication bias in the ego depletion literature and concluded that exercising self-control has no effect on subsequent self-control tasks (Carter, Kofler, Forster, \& McCullough, 2015).

But the bias correction techniques used by Carter et al. (2015) have been called into question. Specifically, the precision-effect test (PET) and precision-effect estimate with standard error (PEESE; Stanley \& Doucouliagos, 2014) have not been thoroughly tested in psychology, and data simulations have revealed that both tests may underestimate effect sizes, often returning estimates no different from zero even when a true effect exists (Inzlicht, Gervais, Berkman, 2015; Moreno et al., 2009; Reed, 2015; Simonsohn, 2017). These overcorrections tend to occur 
in the presence of small samples, heterogeneous effects, and publication bias - characteristics likely to exist in the ego depletion literature.

Additionally, proponents of the resource model have criticized the multi-lab replication study for not actually manipulating ego depletion (Baumeister \& Vohs, 2016a). Specifically, participants in the Hagger et al. (2016) study pressed a computer key in response to words containing the letter $e$, and participants in the depletion condition withheld responding if the $e$ was next to or one letter away from another vowel. Unlike many previous experiments (e.g., Clarkson, Hirt, Jia \& Alexander, 2010; Job, Dweck, Walton, 2010), participants in the Hagger et al. study did not first establish a habit of responding to all es before attempting to follow the more complicated rules. Thus, participants in the multi-lab replication study may not have needed to override a predominant response tendency - a crucial precondition for inducing ego depletion.

Given the impasse between critics and proponents of the resource model, the disputed meta-analytic results, and the lack of high-powered experiments testing the ego depletion effect, we conducted two preregistered experiments with large samples to test again the hypothesis that exercising self-control at Time 1 undermines self-control at Time 2. Specifically, we tested the hypothesis that exercising self-control undermines subsequent attention control.

\section{Ego Depletion and Attention Control}

Attention control is a form of self-control that involves overriding one's predominant attentional tendency. For instance, inhibiting the automatic capture of attention by distractor stimuli or focusing on a particular stimulus for an extended time requires attention control (Schmeichel \& Baumeister, 2010). In both cases, a predominant tendency (i.e., to attend to novel stimuli) must be overridden. 
Previous research has found that attention control is susceptible to ego depletion. For example, participants in one study watched an emotionally-charged movie under instructions to suppress their emotions or to watch the movie as normal. Then they attempted a Stroop task, which involves attention control in the form of responding to non-dominant aspects of a stimulus (font color) and ignoring dominant aspects (word content; Inzlicht \& Gutsell, 2007). Participants who controlled their responses to the movie performed worse on the subsequent Stroop task compared to those who had simply watched the movie, consistent with the idea that exercising self-control at Time 1 impairs attention control at Time 2.

Other self-control challenges, from coping with thoughts of death to suppressing racial prejudices, have also been found to reduce subsequent Stroop performance, resulting in either more errors or slower reaction times (e.g., DeWall, Baumeister, \& Vohs, 2008; Friese \& Wänke, 2014; Gailliot, Schmeichel, \& Baumeister, 2006; Job et al., 2010; Richeson \& Shelton, 2003; Webb \& Sheeran, 2003). Furthermore, performing the Stroop task at Time 1 has been found to reduce subsequent performance (on an anti-saccade task; Dang, Liu, Liu, \& Mao, 2017), which again attests to a role for limited resources in the control of attention, but as discussed earlier the evidentiary value of these and other ego depletion studies have been challenged.

\section{The Process Model of Ego Depletion}

In addition to the empirical challenges posed to the resource model, a new conceptual model has emerged to offer an alternate explanation for the ego depletion effect. According to the process model of ego depletion, exercising self-control does not deplete an inner resource or strength, but rather effortful acts of self-control (because they are aversive events) cause shifts in motivation and attention away from further efforts at self-control and toward signals of rest or reward (Inzlicht \& Schmeichel, 2012; Inzlicht, Schmeichel, \& Macrae, 2014). 
The resource model and the process model make similar predictions in most instances, but their predictions diverge in at least two contexts. First, the resource model assumes that ego depletion influences only subsequent acts of self-control, because the resource is required for the exercise of volition and control and is irrelevant for non-controlled responding. The process model, by contrast, predicts that exercising self-control may influence subsequent motivation and attention even when self-control is not required. Consistent with this view, the aftereffects of self-control have been observed among individuals who simply view emotional pictures without attempting to control their responding (e.g., Finley \& Schmeichel, 2018; Garrison, Crowell, Finley, \& Schmeichel, 2017; Schmeichel, Crowell, \& Harmon-Jones, 2016; see also Crowell, Kelley, \& Schmeichel, 2014; Schmeichel, Harmon-Jones, \& Harmon-Jones, 2010).

Second, and most relevant for the current studies, the process model implies that ego depletion is particularly likely to emerge in the context of emotive (especially rewarding) stimuli. Insofar as exercising self-control causes shifts in motivation and attention toward reward-related stimuli, the ego depletion effect should emerge especially in the context of hedonically positive or otherwise rewarding stimuli. The resource model does not make this prediction because it presumes that ego depletion influences self-control regardless of the emotive characteristics of the self-control task.

To test the hypothesis that ego depletion undermines attention control especially in the context of emotive stimuli, we experimentally manipulated the type of Stroop task participants completed in Experiment 1 of the current investigation. Some participants completed a standard color-word Stroop task as the dependent measure of attention control, whereas other participants completed an emotional Stroop task (i.e., a color-naming task that required participants to ignore the meaning of emotional words as opposed to color words). We assumed that the emotional 
Stroop task, like the standard Stroop task, requires attention control, but the emotional Stroop task entails a more emotive context compared to the standard Stroop task. We thus predicted that Stroop task type would moderate the magnitude of the ego depletion effect, with larger depletion effects on the emotional Stroop, as suggested by the process model of ego depletion. In Experiment 2, all participants completed the same (non-emotive) attention control task as the dependent measure.

\section{The Present Research}

In two experiments we tested the effect of ego depletion on well-established measures of attention control—-the Stroop task (Stroop, 1935) and the Attention Network Test (ANT; Fan, McCandliss, Sommer, Raz, \& Posner, 2002). Ego depletion was induced in each experiment with a writing task that requires some participants to inhibit a predominant writing tendency (see Finley, Crowell, Harmon-Jones, \& Schmeichel, 2017; Mead, Baumeister, Gino, Schweitzer, \& Ariely, 2009; Schmeichel, 2007).

\section{Experiment 1: Ego Depletion and Stroop Performance}

Participants did or did not exert self-control on a writing task before completing either a standard Stroop task or an emotional Stroop task. We included an emotional Stroop task to test the hypothesis that ego depletion would influence attention control particularly in the context of emotive stimuli.

The key prediction was a main effect of prior self-control, such that participants who exerted self-control at Time 1 would perform worse at Time 2 . We also predicted a main effect of Stroop type, such that participants would perform worse on the standard Stroop compared to the emotional Stroop (consistent with prior research; Fox, 1993; Kindt, Bierman, \& Brosschot, 1996; Strauss, Allen, Jorgensen, \& Cramer, 2005). And based on the process model of ego 
depletion we predicted an interaction between prior self-control and Stroop type, such that prior self-control would have a greater influence on emotional Stroop performance. We also predicted moderation by individual differences; see the Supplemental Materials for these analyses, and see our online preregistration for more details (https://osf.io/xktjf/).

\section{Method}

Participants and Design. We planned to conduct the experiment for three semesters with the goal of collecting data from approximately 150 participants per cell, for a total sample of approximately 600 participants. A sample of this size provides $80 \%$ power to detect small-tomedium sized effects $\left(d=0.23\right.$ or $\left.\eta_{p}^{2}=0.013\right)$ for each main effect and the interaction term in the design. We based sample size determination on the power to detect a small-to-medium sized effect because this effect size splits the difference between meta-analytic estimates of the ego depletion effect (see Carter et al., 2015; Hagger, Wood, Stiff, \& Chatzisarantis, 2010).

A total of 667 undergraduate students completed the study in exchange for credit toward a course requirement. Participants were assigned to condition at random in a 2 (Writing Condition: free writing vs. controlled writing $) \times 2$ (Stroop Task: standard Stroop vs. emotional Stroop) between-subjects design. The dependent variable was Stroop performance, operationalized as response interference, error rates, and post-error slowing, respectively. Students participated in groups of 1 to 4 individuals. Due to computer errors $(n=3)$ and experimenter errors $(n=7)$, data from either the Stroop task or the writing task was not recorded, leaving a sample of 657 participants with usable data (397 women; age $M=18.74, S D=1.43 ; 9$ no response).

Procedure. After providing informed consent, participants completed a demographic questionnaire and the self-control manipulation (i.e., the writing task). Participants then 
completed one of two Stroop tasks and personality measures before being debriefed about the purpose of the experiment, thanked, and dismissed. All experimental materials are available online (https://osf.io/hmwr3/).

Self-control manipulation. All participants spent five minutes writing about a recent trip they had taken. Participants in the free writing condition $(n=325)$ were instructed simply to "Write a story about a recent trip you have taken. It may be a trip to the store, to Ohio, or to another country - wherever! Just pick something that is relatively fresh in your memory." Participants in the controlled writing condition $(n=332)$ received an additional instruction: "Very important! Do not use the letters A or N anywhere in your story! If you find yourself writing a word that includes the letters A or N, please stop writing that word and find an alternate way to express your thoughts." Hence, this latter group was required to control their writing by avoiding the use of two common letters, whereas the former group wrote without restrictions.

Stroop task. Participants then performed one of two Stroop tasks on a computer. In the standard Stroop condition $(n=333)$ participants saw the color words red, blue, green, and yellow displayed in red, blue, green, or yellow font and were instructed to respond to the color of the font by pressing keys corresponding to each color. On congruent trials the color word matched the font color, whereas on incongruent trials the color word did not match the font color. In the emotional Stroop condition $(n=324)$ participants saw neutral and emotional words displayed in red, blue, green, or yellow font and were instructed to respond to the color of the font by pressing the keys corresponding to each color. On neutral trials the word was neutral in valence, whereas on emotional trials the word was either positive or negative in valence. The Stroop tasks were run in DirectRT. 
Each Stroop task consisted of four practice trials (participants responded to a string of $X \mathrm{~s}$ displayed in different font colors) followed by three blocks of 40 randomly presented trials, for a total of 120 experimental trials. In the standard Stroop condition each block contained 10 incongruent trials and 30 congruent trials. In the emotional Stroop condition each block contained 10 emotional word trials (5 positive word trials and 5 negative word trials) and 30 neutral word trials. See the Supplemental Materials for the full word list and ratings from the Affective Norms for English Words database (ANEW; Bradley \& Lang, 1999).

Stroop performance can be assessed in multiple ways. As presented in our preregistration document, we examined response interference, quantified as incongruent RTs minus congruent RTs in the standard Stroop condition and emotional RTs minus neutral RTs in the emotional Stroop condition, respectively (correct trials only). We also assessed error rates (i.e., number of errors divided by total number of trials) and post-error slowing (i.e., mean RT on trials after errors minus mean RT on trials before errors) for both Stroop tasks. All RTs are reported in milliseconds. See Table 1 for descriptive statistics of Stroop performance across conditions.

Final questionnaires. Following the Stroop task participants completed questionnaires assessing various aspects of personality including need for cognition (Cacioppo, Petty, \& Kao, 1984), implicit willpower beliefs (Job et al., 2010), behavioral inhibition system and behavioral activation system sensitivity (BIS/BAS; Carver \& White, 1994), action control (Kuhl, 1994), approach and avoidance temperament (Elliot \& Thrash, 2010), and trait self-control (Tangney et al., 2004). Following these questionnaires, participants were fully debriefed about the purpose of the study and dismissed. The entire experiment lasted approximately 45 min.

\section{Results}


We followed a preregistered plan for data analysis (https://osf.io/xktjf/) and conducted additional exploratory analyses. Participants' Stroop data were processed before we calculated performance scores, such that trials with RTs faster than $200 \mathrm{~ms}$ or above or below 3 SDs of a participant's mean RT were dropped. We first analyzed all usable Stroop performance data $(N=$ $657)$, then we conducted the same analyses after applying exclusion criteria $(N=614)$. Note that the preregistered analysis plan assumes the application of the exclusion criteria prior to data analysis.

\section{Stroop Performance}

We analyzed Stroop performance in three separate 2 (Writing Condition: free writing vs. controlled writing $) \times 2$ (Stroop Task: standard vs. emotional) ANOVAs with error rates, response interference, and post-error slowing as the dependent measures.

First, participants made errors at a higher rate in the controlled writing condition $(M=$ $0.064, S D=0.046)$ compared to the free writing condition $(M=0.057, S D=0.040), F(1,653)=$ 4.84, $p=.028, \eta p^{2}=.007, d=0.15,95 \%$ CI $[0.001,0.011]$, supporting the ego depletion hypothesis. Additionally, participants made errors at a higher rate in the standard Stroop task ( $M$ $=0.087, S D=0.036)$ compared to the emotional Stroop task $(M=0.033, S D=0.031), F(1,653)$ $=424.70, p<.001, \eta_{p}^{2}=.394, d=1.61,95 \%$ CI $[0.049,0.059]$. But the Writing Condition $\times$ Stroop Task interaction we had predicted was non-significant, $F(1,653)=0.12, p=.726, \eta_{p}^{2}<$ .001. See Table 1.

Regarding response interference, we did not observe the predicted main effect of writing condition, $F(1,653)<0.001, p=.984, \eta_{p}^{2}<.001, d=0.02,95 \%$ CI $[-10.727,10.504]$. However, the standard Stroop task produced greater interference $(M=160.57, S D=96.76)$ than the emotional Stroop task $(M=0.40, S D=8.81)$, as indicated by a main effect of Stroop task, $F(1$, 
$653)=877.75, p<.001, \eta_{p}{ }^{2}=0.573, d=2.33,95 \%$ CI $[149.552,170.783]$. The Writing

Condition $\times$ Stroop Task interaction, was non-significant, $F(1,653)=0.05, p=.833, \eta_{p}^{2}<.001$. See Table 1.

Post-error slowing could not be calculated for 39 participants because they did not commit enough errors ( $n=36$ committed no errors, and $n=3$ committed only one error in the first or last trial). We observed no significant main effects of Stroop task, $F(1,614)=1.05, p=$ $.306, \eta_{p}^{2}=.002, d=0.09,95 \%$ CI $[-8.748,27.795]$, or writing condition, $F(1,614)=1.29, p=$ $.257, \eta_{p}{ }^{2}=.002, d=0.08,95 \%$ CI $[-28.830,7.713]$, nor any hint of a Writing Condition $\times$ Stroop Task interaction, $F(1,614)=0.06, p=.801, \eta_{p}^{2}<.001$, on post-error slowing.

\section{Analyses following exclusions}

We generated a priori exclusion criteria for the writing task manipulation: failing to follow instructions (e.g., drawing pictures, writing blank spaces instead of $a$ s or $n \mathrm{~s} ; n=7$ ), using more than $3 S D$ s above the mean number of $a$ s and $n \mathrm{~s}$ in the controlled writing task (i.e., use more than 24 as or $n \mathrm{~s} ; n=13$ ), or other experimenter errors resulting in a botched experimental session $(n=2)$. Additionally, although this was not a preregistered exclusion criteria, we excluded data from one participant who reported being colorblind. We also established exclusion criteria regarding Stroop performance. Specifically, we classified as outliers participants scoring more than $3 S D$ s away from the sample mean on response interference $(n=6)$, error rates $(n=7)$, and post-error slowing $(n=7)$, respectively, leaving a sample of 614 participants (374 women; age $M=18.74, S D=1.47,7$ no response).

After exclusions, the results reported above remained unchanged. Most importantly, participants committed errors at a higher rate in controlled writing condition $(M=0.061, S D=$ 0.036) compared to the free writing condition $(M=0.055, S D=0.036)$, as indicated by a 
significant main effect of writing condition, $F(1,610)=5.17, p=.023, \eta_{p}^{2}=.008, d=0.15$,

$95 \%$ CI $[0.001,0.009] .{ }^{1}$

\section{Individual difference moderators}

In the Supplemental Materials we report in full detail the moderation of the ego depletion effect on Stroop performance by individual differences in implicit willpower beliefs, need for cognition, action orientation, and trait self-control, respectively. Briefly, we found no evidence that these individual difference variables moderated the ego depletion effect. In the Supplemental Materials we also report the extent to which individual differences in BIS/BAS and approach/avoidance temperaments moderated the ego depletion effect on emotional Stroop task performance. The only statistically significant effect we observed was an interaction between writing condition and trait BIS, such that higher BIS was associated with stronger response interference to negative emotion words in the free writing condition relative to the controlled writing condition.

\section{Discussion}

Participants who controlled their writing behavior made more errors on subsequent Stroop tasks relative to participants who did not first exercise self-control. Increased error rates are an unambiguous indicator of poorer performance, particularly in the absence of an increase in reaction times (i.e., no speed/accuracy tradeoff), thus Experiment 1 supports the ego depletion effect hypothesized by the resource model of self-control.

\footnotetext{
${ }^{1}$ We conducted additional exploratory analyses (both with and without exclusions) suggested by reviewers. Full details of these analyses can be found in the Supplemental Materials. In brief, we analyzed error rates separately for incongruent/emotional and congruent/neutral trials, respectively, by adding trial type as a within-subjects factor. The interaction between writing condition and trial type was non-significant, indicating that the effect of depletion on error rates was consistent across trial types (both with and without exclusions).
} 
The writing manipulation did not influence RT measures of Stroop performance.

Depleted participants thus made more errors while working at the same rate of speed as nondepleted participants. In all we tested three performance measures (i.e., response interference, error rates, and post-error slowing) because previous research had found that depletion may increase response interference or error rates on attention control tasks (Friese \& Wänke, 2014; Inzlicht \& Gutsell, 2007; Job et al., 2010; Richeson \& Shelton, 2003; Webb \& Sheeran, 2003). Only error rates yielded a depletion effect in the current experiment.

Inspired by the process model of ego depletion we also predicted that the depletion effect would be more prominent in the emotional Stroop condition relative to the standard Stroop condition, but we found no evidence to support this prediction. The emotional Stroop task differs from the standard Stroop task in important ways, but we included both tasks in the current study because both require attention control for successful performance. In the current study (and in some past work; e.g., Fox, 1993; Kindt et al., 1996; Strauss et al., 2005), the standard Stroop elicited more response interference and more errors than the emotional Stroop task did. Indeed, we found no evidence of response interference in the emotional Stroop task. Regardless of which Stroop task participants completed in the current experiment, depleted participants made more errors.

\section{Experiment 2: Ego Depletion and ANT Performance}

Experiment 2 tested the hypothesis that ego depletion undermines attention control on the ANT — a speeded reaction time task that measures distinct aspects of attention (i.e., alerting, orienting, and executive control; Fan et al., 2002). The key prediction was a main effect of depletion such that participants who exerted self-control at Time 1 would perform worse on the ANT at Time 2. We also predicted an interaction between depletion condition and attention 
network, such that ego depletion would undermine especially the executive control network.

Further, we included several individual difference moderators; these analyses are reported in detail in the Supplemental Materials (see our online preregistration, https://osf.io/426uf/). ${ }^{2}$

\section{Method}

Participants and Design. We planned to stop data collection after three semesters with the goal of collecting 150 participants in each condition (300 total). A sample of this size affords $80 \%$ power to detect a small-to-medium sized effect $\left(d=0.32\right.$ or $\left.\eta_{p}^{2}=0.026\right)$. Again, we based sample size determination on the power to detect a small-to-medium sized effect because this effect size splits the difference between meta-analytic estimates of the ego depletion effect (see Carter et al., 2015; Hagger et al., 2010). ${ }^{3}$

A total of 377 undergraduate students completed the study in exchange for credit towards a course requirement. Eighteen participants encountered a computer error or did not complete the ANT, leaving a final sample with usable data of 359 participants (215 women, 1 no response; age $M=18.84, S D=1.07)$.

Procedure. Participants completed the experiment in groups of up to four. After providing informed consent, participants completed demographic questionnaires and received instructions about the writing task. Following the writing task, participants completed the ANT.

Self-control manipulation. The writing task from Experiment 1 served as the manipulation of self-control (see Schmeichel, 2007). Participants were randomly assigned to the

\footnotetext{
2 This hyperlink points to the original preregistration. We updated the page to specify a) sample size determination, and b) our intent to assess median and mean RT and use the mean if the two measures correlated above 0.70 (they did; see Supplemental Materials). These updates (time stamped prior to data analysis) are posted here: https:/osf.io/3vjh5/

${ }^{3}$ We did not base the power analysis for Experiment 2 on the effect sizes observed in Experiment 1 because the two experiments ran concurrently.
} 
free writing condition $(n=186)$ or the controlled writing condition $(n=173)$ and were instructed to write for $5 \mathrm{~min}$.

Attention Network Test. Participants then completed the ANT. The ANT is a cued reaction time task and a variation of the flanker task (Eriksen \& Eriksen, 1974; Posner, 1980). We administered the ANT using a JavaScript program available online (https://www.sacklerinstitute.org/cornell/assays_and_tools/ant/jin.fan/). Participants viewed a row of arrows pointing to the right or left and were instructed to indicate the direction of the central arrow. Congruent trials consisted of 2 flanking arrows on either side of the central arrow pointed in the same direction as the central arrow. Incongruent trials consisted of two flanking arrows on either side of the central arrow pointed in the opposite direction as the central arrow. Neutral trials had only a central arrow (no flankers). The arrow stimuli appeared either above or below a fixation cross and were cued with a warning signal in a number of ways. Specifically, a center cue consisted of an asterisk flashed over the fixation cross to alert participants to the upcoming arrow stimuli, a double cue consisted of two asterisks simultaneously above and below the fixation cross to alert participants to the upcoming stimuli in a larger field of attention, a spatial cue consisted of a single asterisk appearing above or below the fixation and validly cued the location of the upcoming stimuli, and some trials had no cue and only the fixation cross. Thus, the ANT included 12 possible trial types as a function of cue type (center cue, double cue, spatial cue, no cue) and stimulus congruency (congruent, incongruent, neutral). See Fan et al. (2002) for a depiction of this task.

Trials proceeded as follows. A central fixation cross appeared for a random duration between 400-1600 ms, followed by one of the four cue types for $100 \mathrm{~ms}$. The fixation cross remained on screen for another $400 \mathrm{~ms}$ and was followed by arrow stimuli either above or below 
the fixation cross. Participants were instructed to respond as quickly and accurately as possible to the direction of the central arrow target, which remained onscreen until participants responded (up to $1700 \mathrm{~ms}$ ). Participants attempted 24 practice trials and received positive or negative feedback on their responses. Following the practice trials participants completed three blocks of 96 trials each (288 total) with flanker congruency and cue type randomly ordered. The entire task lasted approximately $15 \mathrm{~min}$.

The efficiency of three attention networks is captured by performance as a function of cue type and flanker type. Alerting is calculated by subtracting the mean RT or error rate on double cue trials from mean RT or error rate on no cue trials. Higher scores reflect more efficient alerting. Orienting is calculated by subtracting the mean RT or error rate on spatial cue trials from mean RT or error rate on center cue trials, and higher scores reflect more efficient orienting. Executive control is calculated by subtracting the mean RT or error rate on congruent flanker trials from mean RT or error rate on incongruent flanker trials. Higher scores indicate greater flanker interference and thus less efficient executive control. Error rates were quantified as the number of incorrect responses divided by the total number of trials in which participants responded.

Final questionnaires. Following the ANT participants completed questionnaires assessing various aspects of personality including need for cognition (Cacioppo et al., 1984), implicit willpower beliefs (Job et al., 2010), behavioral inhibition system and behavioral activation system sensitivity (BIS/BAS; Carver \& White, 1994), action control (Kuhl, 1994), approach and avoidance temperament (Elliot \& Thrash, 2010), and trait self-control (Tangney et al., 2004). Following the questionnaires participants were debriefed about the purpose of the study and dismissed. The entire experiment lasted approximately $45 \mathrm{~min}$. 


\section{Results}

We followed a preregistered plan for data analysis (https://osf.io/426uf/) and conducted additional exploratory analyses. Prior to calculating ANT performance the raw RT data were cleaned so that responses faster than $200 \mathrm{~ms}$ and those that fell above or below $2.5 \mathrm{SD}$ from the mean of individuals' own RT distributions were excluded. The three attention network scores are theorized to be independent constructs (Fan et al., 2002). As reported in the Supplemental Materials Table S4, the correlations among attention network scores (both reaction times and error rates) were small, with only alerting and executive control reaction times showing a positive correlation, $r=.166$, and alerting and executive control error rates showing a negative correlation, $r=-.223$. All other correlations were non-significant, $r \mathrm{~s}<|.06|$.

Performance on the ANT was analyzed in two ways. First, we ran 2 (Writing Condition: free writing vs. control) $\times 4$ (Cue Type: center, spatial, double, none) $\times 3$ (Flanker Type: congruent, incongruent, neutral) mixed-model ANOVAs on error rates and RTs (correct trials only) (e.g., Fan et al., 2002; Fan, McCandliss, Fossella, Flombaum, \& Posner, 2005). Second, we analyzed the efficiency of the three attention networks using the subtraction-based scoring procedures described above (see Fan et al., 2002; Fan et al., 2005). When the assumption of sphericity was violated we adjusted degrees of freedom using the Greenhouse-Geisser correction, and when equal variances were not assumed in follow-up tests we corrected degrees of freedom using the Welch-Satterthwaite method. We conducted the preregistered analyses on all participants with usable data $(N=359)$ and then again after applying exclusion criteria $(N=$ 337). Note that the preregistered analysis plan assumes the application of the exclusion criteria prior to data analysis.

\section{ANT Performance}


We observed error rates consistent with the standard ANT effects, with higher error rates on incongruent trials compared to congruent and neutral trials. Error rates were also lowest following spatial cues and (surprisingly) highest following center and double cues. The relevant analyses and descriptive statistics are reported in the Supplemental Materials.

We then tested our core hypothesis regarding the effect of ego depletion on ANT error rates. Supporting our predictions, participants in the controlled writing condition $(M=0.039, S D$ $=0.053)$ made errors at a higher rate compared to participants in the free writing condition $(M=$ 0.026, $S D=0.055), F(1,357)=5.16, p=.024, \eta_{p}^{2}=.014, d=0.24,95 \%$ CI $[0.002,0.023]$. In addition, writing condition interacted with flanker type, $F(1.04,370.00)=5.60, p=.018, \eta_{p}^{2}=$ .015. Specifically, participants in the controlled writing condition $(M=0.103, S D=0.177)$ made errors at a higher rate relative to participants in the free writing condition $(M=0.068, S D=$ 0.087 ) only on incongruent flanker trials, $t(246.82)=2.32, p=.021, d=0.25,95 \%$ CI $[0.005$, 0.064]. Error rates did not differ between the two writing task conditions for congruent trials, $t$ $(357)=0.66, p=.512, d=0.07,95 \% \mathrm{CI}[-0.004,0.010]$, or neutral trials, $t(357)=0.54, p=$ $.592, d=0.06,95 \%$ CI $[-0.005,0.010]$. These results indicate that participants in the controlled writing condition made more errors particularly on ANT trials requiring executive control (i.e., incongruent trials). Writing condition did not interact with cue type, $F(2.71,968.97)=1.27, p=$ $.285, \eta_{p}^{2}=.004$, nor did we find a three-way interaction among writing condition, flanker type, and cue type, $F(4.13,1475.48)=1.58, p=.175, \eta_{p}^{2}=.004$. See Table 2 .

Regarding RTs, we observed standard ANT effects with faster RTs on congruent and neutral flanker trials compared to incongruent flanker trials. Furthermore, spatial cues elicited the fastest RTs and trials with no cues the slowest RTs. The relevant analyses and descriptive statistics are reported in the Supplemental Materials. 
Then, we tested the main ego depletion hypothesis on ANT RTs. Writing condition did not exert a main effect on RTs, $F(1,353)=0.098, p=.754,95 \%$ CI $[-17.85,12.94]$. Writing condition also did not interact with flanker type, $F(1.48,522.30)=0.32, p=.661, \eta_{p}^{2}=.001$, or cue type, $F(2.66,937.35)=1.21, p=.305, \eta_{p}^{2}=.003$, nor was there a three-way interaction among writing condition, flanker type, and cue type, $F(5.28,1863.65)=0.96, p=.444, \eta_{p}^{2}=$ .003. Hence, the writing manipulation did not influence RTs on any aspect of the ANT. See Table 2.

Next, we calculated the three attention network scores based on the subtractions outlined above. The descriptive statistics of the three attention network scores and the correlations among them are reported in the Supplemental Materials.

We ran a multivariate analysis (MANOVA) with writing condition as the independent variable and each attention network score as the dependent variables. ${ }^{4}$ For attention network scores based on error rates, the multivariate effect of writing condition was significant, $F(3,355)$ $=4.36, p=.005, \eta_{p}^{2}=.035$. For attention network scores based on RTs, the multivariate effect of writing condition on attention network scores was non-significant, $F(3,354)=0.244, p=.865$, $\eta_{p}^{2}=.002$

Follow-up univariate tests on error rates revealed that participants in the controlled writing condition $(M=.091, S D=.167)$ had higher executive control network scores compared to participants in the free writing condition $(M=.060, S D=.075), F(1,357)=5.12, p=.024$,

\footnotetext{
${ }^{4}$ We preregistered three independent $t$-tests of the effect of writing condition on each ANT score. After completing the study, but before we analyzed the data, we decided to conduct a single multivariate ANOVA including all ANT scores as dependent variables. Results do not change using $t$-tests for attention network RT scores: alerting, $t(357)=0.32, p=.747$; orienting, $t(357)=0.29, p=.773$; executive control, $t(356)=-0.74, p=.461$; and attention network error rate scores: alerting, $t(357)=0.65, p=.518$, orienting, $t(357)=2.33, p=.020$, and executive control, $t(234.01)=2.21, p=.028$.
} 
$\eta_{p}^{2}=.014, d=0.24,95 \%$ CI $[0.004,0.057]$, indicating greater flanker interference under ego depletion. Participants in the controlled writing condition $(M=.010, S D=.026)$ also had higher orienting scores compared to the free writing condition $(M=.004, S D=.024), F(1,357)=5.45$, $p=.020, \eta_{p}^{2}=.015, d=0.25,95 \% \mathrm{CI}[0.001,0.011]$, indicating that participants in the controlled writing condition had more efficient (accurate) orienting compared to participants in the free writing condition. Alerting network scores did not differ between the two writing conditions, $F(1,357)=0.42, p=.518, \eta_{p}^{2}=.001, d=0.07,95 \%$ CI $[-0.004,0.008]$.

Thus, compared to the free writing condition, completing the controlled writing task increased the number of errors on incongruent relative to congruent trials, thereby reflecting reduced error efficiency in the executive control network, and increased the number of errors on center cue relative to spatial cue trials, thereby reflecting increased error efficiency in the orienting network.

\section{Analysis following exclusions}

We generated several a priori exclusion criteria for participants who completed the study and did not experience computer errors: failure to follow writing task instructions (e.g., drawing, writing blank spaces instead of the letters $a$ or $n$ or using more than $3 S D$ from the group mean of as or $n \mathrm{~s} ; n=8$ ), and being a statistical outlier (more than $3 S D$ from the sample mean) on total ANT trials completed $(n=5)$, grand mean RT $(n=4)$, and number of errors $(n=10)$, respectively. After these exclusions we had a sample of 337 participants (controlled writing $n=$ 156, free writing $n=181)$.

When we excluded participants based on the specified criteria, the effects of writing condition changed from the findings reported above. Specifically, the main effect of writing task on error rates became non-significant, $F(1,335)=1.42, p=.235, \eta_{p}^{2}=.004, d=0.14,95 \%$ CI [- 
0.002, 0.010], and the Writing Task $\times$ Flanker Type interaction also became non-significant, $F$ $(1.05,350.85)=0.69, p=.413, \eta_{p}^{2}=.002$. In addition, the multivariate effect of writing condition on attention network scores based on error rates became non-significant, $F(3,333)=$ $1.70, p=.166, \eta_{p}^{2}=.015$. Hence, following the exclusions of outliers, the effects of the ego depletion manipulation changed such that the writing task no longer influenced error rates.

\section{Exploratory Analyses}

Given that the effects of writing condition became non-significant when excluding outliers, we explored whether exclusion rates differed across conditions. We conducted chisquare tests on the frequencies of exclusions for having outlying values on the dependent measure (total trials completed, RTs, and error rates) as a function of writing condition. Altogether, $2.7 \%$ of participants in the free writing condition and $5.8 \%$ of participants in the controlled writing condition were excluded from analyses for being outliers on some aspect of ANT performance; this difference was not statically significant, $x^{2}(1)=2.14, p=.143$. Regarding the total number of trials completed, $1.1 \%$ of participants in the free writing condition and $1.7 \%$ of participants in the controlled writing condition were excluded; this difference was not significant, $x^{2}(1)=0.28, p=.595$. Regarding RTs, $1.6 \%$ of participants in the free writing condition and $0.6 \%$ of participants in the controlled writing condition were excluded; this difference was also not significant, $x^{2}(1)=0.87, p=.351$.

Regarding error rates, we observed that $1.1 \%$ of participants in the free writing condition and $4.6 \%$ of participants in the controlled writing condition were excluded, and this difference was statistically significant, $x^{2}(1)=4.17, p=.041$. Hence, participants committed errors at an inordinately high rate more often following the controlled writing task relative to the free writing task. When these outliers were included in the analyses, the effect of writing condition on error 
rates was statistically significant. The effect became non-significant when these outliers were excluded. $^{5}$

\section{Individual difference moderators}

In the Supplemental Materials we report in detail analyses testing the moderation of the ego depletion effect by individual differences in implicit willpower beliefs, need for cognition, action orientation, BIS/BAS, approach/avoidance temperaments, and trait self-control, respectively. A few significant effects emerged. Briefly, individuals higher (versus lower) in action orientation (failure subscale; AOF) showed a bigger ego depletion effect on executive control error rates. Individuals higher in action orientation (decision subscale; AOD) also had slower reaction times in the free writing condition, but this relationship disappeared in the controlled writing condition. Individuals higher (versus lower) in AOD also showed a bigger ego depletion effect on executive control error rates. And individuals higher (versus lower) on action orientation (performance subscale; AOP) showed a bigger depletion effect on orienting RTs. Lastly, regarding trait self-control, we found individuals higher (versus lower) in trait selfcontrol showed a bigger ego depletion effect on executive control error rates.

\section{Discussion}

Participants who controlled their writing behavior at Time 1 made more errors on the ANT at Time 2, relative to participants who did not exercise self-control on the initial writing task. This was true particularly when ANT trials required executive control (i.e., on incongruent flanker trials). Thus, Experiment 2 yielded additional support for the ego depletion effect. As in Experiment 1, the RT measures of performance were unaffected by the writing manipulation;

\footnotetext{
${ }^{5}$ We conducted parallel chi-square tests on frequency of being excluded for outlying values on the dependent measures in Study 1 and found no effect of condition. See Supplemental Materials.
} 
only error rates increased under depletion. Participants who had previously exercised self-control thus worked at the same rate of speed but made more errors than other participants (i.e., no speed/accuracy tradeoff) — a clear indication of poorer performance after exercising self-control.

A handful of participants made an inordinate number of errors (more than $3 S D$ s above the sample mean) on the ANT. We found that the writing manipulation increased error rates in Experiment 2 when we analyzed all usable data (including the outliers), but the effect was reduced to non-significance following the exclusion of participants with very high error rates. Our exploratory analysis revealed that participants in the controlled writing condition were more likely to be excluded for making numerous errors. Although we had not anticipated differential exclusion rates as a function of experimental condition, the pattern is congruent with the resource model: Participants who previously exercised self-control performed worse.

\section{Mini Meta-Analysis: Estimating the Effect Size of Depletion on Error Rates}

We estimated the effect of ego depletion on attention control errors across the two experiments to clarify the extent to which the effect hinges on the exclusion of outliers.

\section{Methods}

Participants and Analysis Plan. We extracted effect sizes for the effect of writing task on error rates on the two Stroop tasks from Experiment 1 and the ANT from Experiment 2. We used the meta-analytic approach described by Goh, Hall, and Rosenthal (2016; additional details at https://osf.io/95mcn/).

\section{Results}

Across the full sample $(N=1,016)$ we meta-analyzed the three effects using a fixed effects approach. Participants in the controlled writing condition committed errors at higher rates compared to participants in the free writing condition, $d=0.20,95 \% \mathrm{CI}[0.07,0.32], Z=3.12, p$ 
$=.002$. The depletion effect was not moderated by task type; contrast analyses found nonsignificant differences in the depletion effect on the ANT versus the standard Stroop task, $Z=$ $0.654, p=.513$, the standard Stroop versus the emotional Stroop task, $Z=-0.510, p=.610$, and the ANT versus the emotional Stroop task, $Z=-0.130, p=.897$. The overall depletion effect did not change when we used a random effects approach, $d=0.20$, and a one-sample $t$ test revealed that the depletion effect was significantly different from zero, $t(2)=6.55, p=.023$.

Following exclusions based on our preregistered criteria $(N=951)$, the weighted mean effect of depletion on error rates remained statistically significant, $d=0.16,95 \%$ CI $[0.04,0.30]$, $Z=2.57, p=.010$. The depletion effect again was not moderated by task type; contrast analyses revealed non-significant differences in the depletion effect on the ANT versus the standard Stroop task, $Z=0.252, p=.801$, the standard Stroop versus the emotional Stroop task, $Z=0, p>$ .999 , and the ANT versus the emotional Stroop task, $Z=0.252, p=.801$. Using a random effects approach did not change the overall depletion effect size estimate, $d=0.16$, and a one-sample $t$ test again revealed that the depletion effect was significantly different from zero, $t(2)=12.5, p=$ .006.

\section{General Discussion}

Two preregistered experiments found that exercising self-control on a writing task at Time 1 increases errors on tests of attention control at Time 2. These results are consistent with the ego depletion effect and support the resource model of self-control.

The depletion manipulation affected errors but not RT measures in both experiments. Both RTs and errors are indicators of performance, and in past research ego depletion has sometimes increased errors and sometimes increased RTs. We were agnostic about which performance measure would be most affected in the current experiments (theoretical accounts of 
ego depletion do not point to one or the other as more susceptible to the ego depletion effect), but the data pointed to increased errors. The fact that RTs were unaffected suggests that depleted participants did not shift to a speedier but more error prone response mode (i.e., no speed/accuracy tradeoff); they simply made more mistakes working at the same speed as nondepleted participants. This pattern represents clear evidence for poorer attention control under ego depletion.

To be sure, the most powerful evidence for ego depletion would have entailed slower response times, more errors, and reduced post-error slowing. This combination of results would have revealed a rather devastating combination of effects of ego depletion on performance. We found a more modest effect in the form of increased errors only. This pattern of results is congruent with prior evidence that depletion affects response times or errors, but not both. One could argue that errors are the purest indicator of poor self-control insofar as they reveal literal failures to inhibit a predominant response tendency, as opposed to response time measures, which would indicate slower but successful inhibition. The absence of a depletion effect on posterror slowing suggests that depleted participants, despite making more errors, regulated their responding adaptively following those errors. Hence, we found that depletion undermined but did not devastate attention control.

We assessed the depletion effect among all participants with usable data and among somewhat smaller samples following exclusions. In Experiment 1, the effect on error rates was plain in both analyses. In Experiment 2, the effect was statistically significant only when analyzing all usable data. But then, our exploratory analyses revealed that depleted (versus nondepleted) participants in Experiment 2 were more likely to be excluded for making numerous errors - as the resource model would predict - so perhaps it is not surprising that the depletion 
effect became weaker when highly error-prone participants were excluded. Because exclusions for making errors differed, unexpectedly, as a function of experimental condition in Experiment 2, we conducted and reported our analyses both with and without exclusions.

The mini meta-analysis provides the most accurate estimate of the depletion effect on attention control. The effect of depletion on error rates was significant, with $d$ s ranging from 0.16 (with exclusions) to 0.20 (without exclusions). These effect size estimates are smaller than the initial meta-analysis of the depletion effect $(d=0.62$; Hagger et al., 2010), but larger than the subsequent meta-analysis that bias-corrected the effect to zero (Carter et al., 2015). Based on the current experiments, we conclude that the "truth" is somewhere in between-depletion has a small but significant effect on attention control.

We had predicted that ego depletion would exert a stronger influence on emotional Stroop performance compared to standard Stroop performance in Experiment 1, based on the process model of depletion (Inzlicht \& Schmeichel, 2012), but found no evidence to support this prediction. Regardless of which Stroop task participants completed in Experiment 1, depleted participants made more errors. For Experiment 2 we had hypothesized that depletion would be especially consequential for the executive attention network, and the error results supported this prediction. But we also found that depletion increases efficiency in the orienting network. This unexpected finding is not consistent with the resource model and needs to be replicated.

\section{Limitations and Constraints on Generality}

The current experiments are not without limitations. We used only one 5-minute manipulation of ego depletion. Presumably, shorter or easier manipulations would result in smaller or null effects, whereas longer or more challenging manipulations would exert even larger effects (e.g., Sjåstad, \& Baumeister, 2018; Vohs, Baumeister, \& Schmeichel, 2012). 
Further, we found smaller effects in Experiment 2 when we excluded some participants from analyses, but more participants were excluded from one condition than the other. This pattern serves as a reminder that care be taken to establish exclusion criteria that do not operate differently as a function of experimental conditions or other controllable factors. Based on our experience with the current experiments, we encourage researchers to preregister their intentions to test hypotheses both before and after applying exclusion criteria and then report and consider differences in the results (if any) across both analyses.

In both experiments we tried to find evidence for moderation of the depletion effect by trait self-control and related individual differences, but we did not find much (or consistent) evidence for moderation. These null findings may be due in part to the fact that the current studies, although well powered to detect main effects, had less statistical power to detect moderation by individual differences. Given the modest magnitude of the depletion effects observed in the current experiments, even greater statistical power (including a larger sample of participants) would be required to find moderation by individual difference variables.

We did not include manipulation checks in the current experiments because past research had convinced us they were not necessary. The controlled writing task has been used many times in previous research and has routinely been observed to be more difficult and to require more self-control than the free writing task. Nonetheless, it is likely that some participants found the controlled writing task to be more effortful or challenging than did others. Presumably, participants who exerted more effort on the task or experienced more fatigue as a result of performing the task would show stronger aftereffects (e.g., Dang et al., 2017). Going forward, we encourage researchers to assess subjective exertion and fatigue in experiments on ego depletion, both to verify that the depletion manipulation worked and to permit a test of the 
depletion effect among those who experienced the manipulation as more (versus less) demanding.

Based on the process model of self-control we had predicted a stronger influence of ego depletion on emotional Stroop versus standard Stroop performance. We found no support for this prediction, which casts doubt on the process model prediction. But the null finding (like most null findings) is ambiguous. In the current research the emotional Stroop task engendered little or no response interference. The Stroop type manipulation thus failed to manipulate emotional context on an attention control task in the manner we had intended, which in turn hindered our ability to test the process model prediction. At best it is unclear if the null interaction effect between depletion condition and Stroop type was due to the incorrectness of the prediction or to the insufficiency of the emotional Stroop task. Future research examining the aftereffects of selfcontrol in more emotional (especially tempting or rewarding) contexts should provide a clearer test of the process model predictions.

These experiments tested only US college students, so the extent to which the results generalize (e.g., to older individuals, non-Westerners) remains an open question. Some evidence suggests that ego depletion operates differently in other cultural contexts (Savani \& Job, 2017). We also cannot extrapolate from the current laboratory findings to more naturalistic contexts, so the effects of ego depletion in the wild remain an avenue for additional research (e.g., Hofmann, Baumeister, Foerster, \& Vohs, 2012). On the other hand, we found similar effects of ego depletion on error rates across two distinct dependent measures (i.e., Stroop tasks and the ANT), which lends credence to the conclusion that depletion undermines success at attention control.

\section{Conclusion}


Ego depletion has often been tested in small samples of participants, the depletion literature is almost certainly marked by publication bias, and meta-analyses have yielded divergent effect size estimates. The existence of ego depletion has thus been challenged. Given the controversy surrounding ego depletion, we believe it is crucial to test the effect in large, preregistered experiments. The preregistered methods, hypotheses, and analysis plans associated with the current experiments reduced flexibility in data analysis (e.g., p-hacking) and increased transparency, and both experiments included large samples, thereby lending confidence to the results.

The evidence for an ego depletion effect on attention control reported here should encourage explorations into mechanisms driving the effect. For instance, does depletion result from a drop in resources (Muraven \& Baumeister, 2000), a strategic conservation of resources (Baumeister \& Vohs, 2016b; Beedie \& Lane, 2012), or a motivated shift away from have-to goals toward want-to goals (Inzlicht, Schmiechel, \& Macrae, 2014)? The answer remains to be seen, but the current experiments provide new evidence that this is still a question worth asking. 


\section{References}

Baumeister, R. F., \& Vohs, K. D. (2016a). Misguided effort with elusive implications. Perspectives on Psychological Science, 11, 574-575.

Baumeister, R. F., \& Vohs, K. D. (2016b). Strength model of self-regulation as limited resource: Assessment, controversies, updates. In J. Olson and M. Zanna (Eds.), Advances in Experimental Social Psychology (Vol. 54) (pp. 67-127). Cambridge, MA: Academic Press.

Beedie, C. J., \& Lane, A. M. (2012). The role of glucose in self-control: Another look at the evidence and an alternative conceptualization. Personality and Social Psychology Review, 16, 143-153.

Bradley, M. M., \& Lang, P. J. (1999). Affective norms for English words (ANEW): Instruction manual and affective ratings (pp. 1-45). Technical Report C-1, The Center for Research in Psychophysiology, University of Florida.

Cacioppo, J. T., Petty, R. E., \& Kao, C. F. (1984). The efficient assessment of need for cognition. Journal of Personality Assessment, 48, 306-307.

Carter, E. C., Kofler, L. M., Forster, D. E., \& McCullough, M. E. (2015). A series of metaanalytic tests of the depletion effect: Self-control does not seem to rely on a limited resource. Journal of Experimental Psychology: General, 144, 796-815.

Carver, C. S., \& White, T. L. (1994). Behavioral inhibition, behavioral activation, and affective responses to impending reward and punishment: The BIS/BAS Scales. Journal of Personality and Social Psychology, 67, 319-333.

Crowell, A., Kelley, N. J., \& Schmeichel, B. J. (2014). Trait approach motivation moderates the aftereffects of self-control. Frontiers in Psychology, 5: 1112. 
Dang, J., Liu, Y., Liu, X., \& Mao, L. (2017). The ego could be depleted, providing initial exertion is depleting: A preregistered experiment of the ego depletion effect. Social Psychology, 48, 242-245.

Clarkson, J. J., Hirt, E. R., Jia, L., \& Alexander, M. B. (2010). When perception is more than reality: The effects of perceived versus actual resource depletion on self-regulatory behavior. Journal of Personality and Social Psychology, 98, 29.

DeWall, C. N., Baumeister, R. F., \& Vohs, K. D. (2008). Satiated with belongingness? Effects of acceptance, rejection, and task framing on self-regulatory performance. Journal of Personality and Social Psychology, 95, 1367.

Elliot, A. J., \& Thrash, T. M. (2010). Approach and avoidance temperament as basic dimensions of personality. Journal of Personality, 78, 865-906.

Eriksen, B. A., \& Eriksen, C. W. (1974). Effects of noise letters upon the identification of a target letter in a nonsearch task. Attention, Perception, \& Psychophysics, 16, 143-149.

Fan, J., McCandliss, B. D., Fossella, J., Flombaum, J. I., \& Posner, M. I. (2005). The activation of attentional networks. NeuroImage, 26, 471-479.

Fan, J., McCandliss, B. D., Sommer, T., Raz, A., \& Posner, M. I. (2002). Testing the efficiency and independence of attentional networks. Journal of Cognitive Neuroscience, 14, 340347.

Finley, A. J., Crowell, A. L., Harmon-Jones, E., \& Schmeichel, B. J. (2017). The influence of agreeableness and ego depletion on emotional responding. Journal of personality, 85, 643-657.

Finley, A. J., \& Schmeichel, B. J. (2018). Aftereffects of self-control on positive emotional reactivity. Manuscript submitted for publication. 
Fox, E. (1993). Attentional bias in anxiety: Selective or not? Behaviour Research and Therapy, 31, 487-493.

Friese, M., \& Wänke, M. (2014). Personal prayer buffers self-control depletion. Journal of Experimental Social Psychology, 51, 56-59.

Gailliot, M. T., Schmeichel, B. J., \& Baumeister, R. F. (2006). Self-regulatory processes defend against the threat of death: Effects of self-control depletion and trait self-control on thoughts and fears of dying. Journal of Personality and Social Psychology, 91, 49-62.

Garrison, K. E., Crowell, A. L., Finley, A. J., \& Schmeichel, B. J. (2017). Effects of prior mental effort on picture processing: An ERP investigation. Psychophysiology, 54, 1714-1725.

Goh, J. X., Hall, J., Rosenthal, R. (2016). Mini meta-analysis of your own studies: Some arguments on why and a primer on how. Social and Personality Psychology Compass, $10,535-549$.

Hagger, M. S., Chatzisarantis, N. L., Alberts, H., Anggono, C. O., Batailler, C., Birt, A. R., ... \& Calvillo, D. P. (2016). A multilab preregistered replication of the ego-depletion effect. Perspectives on Psychological Science, 11, 546-573.

Hagger, M. S., Wood, C., Stiff, C., \& Chatzisarantis, N. L. (2010). Ego depletion and the strength model of self-control: a meta-analysis. Psychological Bulletin, 136, 495-525.

Hofmann, W., Baumeister, R. F., Foerster, G., \& Vohs, K. D. (2012). Everyday temptations: An experience sampling study of desire, conflict, and self-control. Journal of Personality and Social Psychology, 102, 1318-1335.

Inzlicht, M., \& Gutsell, J. N. (2007). Running on empty: Neural signals for self-control failure. Psychological Science, 18, 933-937. 
Inzlicht, M., Gervais, W., \& Berkman, E. (2015). Bias-correction techniques alone cannot determine whether ego depletion is different from zero: Commentary on Carter, Kofler, Forster, \& McCullough, 2015. Available at SSRN: https://ssrn.com/abstract=2659409 or http://dx.doi.org/10.2139/ssrn.2659409

Inzlicht, M., \& Schmeichel, B. J. (2012). What is ego depletion? Toward a mechanistic revision of the resource model of self-control. Perspectives on Psychological Science, 7, 450-463.

Inzlicht, M., Schmeichel, B. J., \& Macrae, C. N. (2014). Why self-control seems (but may not be) limited. Trends in Cognitive Sciences, 18, 127-133.

Job, V., Dweck, C. S., \& Walton, G. M. (2010). Ego depletion-Is it all in your head? Implicit theories about willpower affect self-regulation. Psychological Science, 21, 1686-1693.

Kindt, M., Bierman, D., \& Brosschot, J. F. (1996). Stroop versus Stroop: Comparison of a card format and a single-trial format of the standard color-word Stroop task and the emotional Stroop task. Personality and Individual Differences, 21, 653-661.

Kuhl, J. (1994). Action versus state orientation: Psychometric properties of the action control scale (ACS-90). In J. Kuhl and J. Beckmann (Eds.), Volition and personality: Action versus state orientation, (pp 47-59). Seattle, WA: Hogrefe \& Huber.

Mead, N. L., Baumeister, R. F., Gino, F., Schweitzer, M. E., \& Ariely, D. (2009). Too tired to tell the truth: Self-control resource depletion and dishonesty. Journal of experimental social psychology, 45, 594-597.

Mischel, W., Shoda, Y., \& Peake, P. K. (1988). The nature of adolescent competencies predicted by preschool delay of gratification. Journal of Personality and Social Psychology, 54, 687. 
Moreno, S. G., Sutton, A. J., Ades, A. E., Stanley, T. D., Abrams, K. R., Peters, J. L., \& Cooper, N. J. (2009). Assessment of regression-based methods to adjust for publication bias through a comprehensive simulation study. BMC Medical Research Methodology, 9, 2. doi:10.1186/1471-2288-9-2

Muraven, M., \& Baumeister, R. F. (2000). Self-regulation and depletion of limited resources: Does self-control resemble a muscle? Psychological bulletin, 126, 247.

Posner, M. I. (1980). Orienting of attention. Quarterly Journal of Experimental Psychology, 32, $3-25$.

Reed, W. R. (2015). A Monte Carlo analysis of alternative meta-analysis estimators in the presence of publication bias. Economics: The Open-Access, Open-Assessment E-Journal, 9 (2015-30): 1-40. http://dx.doi.org/10.5018/economics-ejournal.ja.2015-30

Richeson, J. A., \& Shelton, J. N. (2003). When prejudice does not pay: Effects of interracial contact on executive function. Psychological Science, 14, 287-290.

Savani, K., \& Job, V. (2017). Reverse ego-depletion: Acts of self-control can improve subsequent performance in Indian cultural contexts. Journal of Personality and Social Psychology, 113, 589-607.

Schmeichel, B. J. (2007). Attention control, memory updating, and emotion regulation temporarily reduce the capacity for executive control. Journal of Experimental Psychology: General, 136, 241.

Schmeichel, B. J., \& Baumeister, R. F. (2010). Effortful attention control. In B. Bruya (Ed.), Effortless attention: A new perspective in the cognitive science of attention and action (pp. 29-49). Cambridge, MA: MIT Press. 
Schmeichel, B. J., Crowell, A., \& Harmon-Jones, E. (2015). Exercising self-control increases relative left frontal cortical activation. Social Cognitive and Affective Neuroscience, 11, 282-288.

Schmeichel, B. J., Harmon-Jones, C., \& Harmon-Jones, E. (2010). Exercising self-control increases approach motivation. Journal of Personality and Social Psychology, 99, 162173.

Simonsohn, U. (2017, April 12). PET-PEESE is not like homeopathy [Web log comment]. Retrieved from http://datacolada.org/59

Sjåstad, H., \& Baumeister, R. F. (2018). The future and the will: Planning requires self-control, and ego depletion leads to planning aversion. Journal of Experimental Social Psychology, $76,127-141$.

Stanley, T. D., \& Doucouliagos, H. (2014). Meta-regression approximations to reduce publication selection bias. Research Synthesis Methods, 5, 60-78.

Strauss, G. P., Allen, D. N., Jorgensen, M. L., \& Cramer, S. L. (2005). Test-retest reliability of standard and emotional Stroop tasks: An investigation of color-word and picture-word versions. Assessment, 12, 330-337.

Stroop, J. R. (1935). Studies of interference in serial verbal reactions. Journal of Experimental Psychology, 18, 643-662.

Tangney, J. P., Baumeister, R. F., \& Boone, A. L. (2004). High self-control predicts good adjustment, less pathology, better grades, and interpersonal success. Journal of Personality, 72, 271-324. 
Vohs, K. D., Baumeister, R. F., \& Schmeichel, B. J. (2012). Motivation, personal beliefs, and limited resources all contribute to self-control. Journal of Experimental Social Psychology, 48, 943-947.

Webb, T. L., \& Sheeran, P. (2003). Can implementation intentions help to overcome egodepletion? Journal of Experimental Social Psychology, 39, 279-286. 
Table 1

Performance as a Function of Stroop Type and Writing Condition (Experiment 1)

Free Writing

Controlled Writing

\begin{tabular}{lrcccccc} 
Trial Type & & Standard & Emotional & Overall & Standard & Emotional & Overall \\
\hline Congruent/ & RT & 691.68 & 657.59 & & 680.97 & 661.23 & \\
Neutral & & $(116.15)$ & $(86.17)$ & & $(109.87)$ & $(153.20)$ & \\
& Error rate & 0.023 & 0.031 & & 0.026 & 0.037 & \\
& & $(0.029)$ & $(0.027)$ & & $(0.039)$ & $(0.036)$ & \\
Incongruent/ & RT & 851.73 & 661.60 & & 842.04 & 660.24 & \\
Emotional & & $(175.34)$ & $(89.01)$ & & $(163.98)$ & $(138.75)$ & \\
& Error rate & 0.271 & 0.027 & & 0.279 & 0.036 & \\
& & $(0.069)$ & $(0.033)$ & & $(0.064)$ & $(0.049)$ & \\
Totals & RT & 160.04 & 1.02 & 80.78 & 161.08 & -0.23 & 82.37 \\
& Interference & $(102.56)$ & $(7.39)$ & $(107.83)$ & $(91.17)$ & $(10.02)$ & $(103.99)$ \\
& Error rate & 0.085 & 0.030 & 0.057 & 0.090 & 0.036 & 0.064 \\
& & $(0.032)$ & $(0.025)$ & $(0.040)$ & $(0.039)$ & $(0.036)$ & $(0.046)$ \\
& Post Error & 9.62 & 2.44 & 6.34 & 1.40 & -10.46 & -4.12 \\
& Slowing & $(83.51)$ & $(130.28)$ & $(107.29)$ & $(89.94)$ & $(150.94)$ & $(122.11)$ \\
\hline
\end{tabular}

Note: Values represent $M$ s and SDs. Cell sizes are as follows: $n=163$ in the free writing plus standard Stroop condition; $n=162$ in the free writing plus emotional Stroop condition; $n=170$ in the controlled writing plus standard Stroop condition; $n=162$ in the controlled writing plus emotional Stroop condition. 
Table 2

ANT Performance as a Function of Cue Type and Writing Condition (Experiment 2)

Free Writing

\begin{tabular}{|c|c|c|c|c|c|c|c|}
\hline Cue & & Neutral & Congruent & Incongruent & Neutral & Congruent & Incongruent \\
\hline \multirow[t]{2}{*}{ None } & RT & $\begin{array}{l}560.68 \\
(81.87)\end{array}$ & $\begin{array}{l}602.25 \\
(91.65)\end{array}$ & $\begin{array}{l}700.20 \\
(94.57)\end{array}$ & $\begin{array}{l}561.09 \\
(65.55)\end{array}$ & $\begin{array}{l}597.58 \\
(73.39)\end{array}$ & $\begin{array}{l}702.99 \\
(91.07)\end{array}$ \\
\hline & $\begin{array}{r}\text { Error } \\
\text { rate }\end{array}$ & $\begin{array}{c}0.010 \\
(0.041)\end{array}$ & $\begin{array}{c}0.000 \\
(0.000)\end{array}$ & $\begin{array}{c}0.062 \\
(0.089)\end{array}$ & $\begin{array}{c}0.011 \\
(0.029)\end{array}$ & $\begin{array}{c}0.000 \\
(0.000)\end{array}$ & $\begin{array}{c}0.095 \\
(0.179)\end{array}$ \\
\hline \multirow[t]{2}{*}{ Center } & RT & $\begin{array}{l}510.58 \\
(76.92)\end{array}$ & $\begin{array}{l}561.83 \\
(91.17)\end{array}$ & $\begin{array}{l}688.58 \\
(95.84)\end{array}$ & $\begin{array}{l}506.63 \\
(64.44)\end{array}$ & $\begin{array}{l}556.28 \\
(71.40)\end{array}$ & $\begin{array}{l}685.79 \\
(86.47)\end{array}$ \\
\hline & $\begin{array}{r}\text { Error } \\
\text { rate }\end{array}$ & $\begin{array}{c}0.009 \\
(0.037)\end{array}$ & $\begin{array}{c}0.000 \\
(0.000)\end{array}$ & $\begin{array}{c}0.070 \\
(0.090)\end{array}$ & $\begin{array}{c}0.012 \\
(0.034)\end{array}$ & $\begin{array}{c}0.000 \\
(0.000)\end{array}$ & $\begin{array}{c}0.115 \\
(0.183)\end{array}$ \\
\hline \multirow[t]{2}{*}{ Double } & RT & $\begin{array}{l}498.79 \\
(68.76)\end{array}$ & $\begin{array}{l}550.77 \\
(78.12)\end{array}$ & $\begin{array}{c}677.70 \\
(102.88)\end{array}$ & $\begin{array}{l}501.54 \\
(62.47)\end{array}$ & $\begin{array}{l}547.77 \\
(67.62)\end{array}$ & $\begin{array}{l}675.50 \\
(84.86)\end{array}$ \\
\hline & $\begin{array}{r}\text { Error } \\
\text { rate }\end{array}$ & $\begin{array}{c}0.011 \\
(0.033)\end{array}$ & $\begin{array}{c}0.006 \\
(0.042)\end{array}$ & $\begin{array}{c}0.085 \\
(0.116)\end{array}$ & $\begin{array}{c}0.010 \\
(0.027)\end{array}$ & $\begin{array}{c}0.011 \\
(0.040)\end{array}$ & $\begin{array}{c}0.114 \\
(0.188)\end{array}$ \\
\hline \multirow[t]{2}{*}{ Spatial } & RT & $\begin{array}{l}479.39 \\
(73.71)\end{array}$ & $\begin{array}{l}523.94 \\
(82.31)\end{array}$ & $\begin{array}{l}632.73 \\
(85.40)\end{array}$ & $\begin{array}{l}472.60 \\
(58.01)\end{array}$ & $\begin{array}{l}520.24 \\
(66.30)\end{array}$ & $\begin{array}{l}629.99 \\
(83.76)\end{array}$ \\
\hline & $\begin{array}{r}\text { Error } \\
\text { rate }\end{array}$ & $\begin{array}{c}0.009 \\
(0.038)\end{array}$ & $\begin{array}{c}0.000 \\
(0.000)\end{array}$ & $\begin{array}{c}0.056 \\
(0.091)\end{array}$ & $\begin{array}{c}0.012 \\
(0.033)\end{array}$ & $\begin{array}{c}0.000 \\
(0.000)\end{array}$ & $\begin{array}{c}0.087 \\
(0.185)\end{array}$ \\
\hline
\end{tabular}

